\title{
Contre les nouveaux féodalismes
}

\author{
Christian Vandendorpe
}

Université d’Ottawa

Le Débat, no 117, novembre 2001, p. 53-58.

Il était aussi improbable qu'un avocat du Barreau endosse mes propositions [Pour une bibliothèque virtuelle universelle] que de voir des dirigeants de Microsoft faire l'apologie du logiciel libre. Tout comme ces derniers, Me Pierrat semble étrangement sur la défensive si l'on en juge par le recours massif à la raillerie et aux sous-entendus qui obscurcissent son argumentation. Ses comparaisons sont éloquentes à cet égard. La proposition de donner libre accès aux ouvres sur le Web après un délai de cinq ou dix ans est ainsi assimilée à la "distribution de fusils-mitrailleurs dans la rue, au prétexte qu'ils ont grandement amélioré les conditions du braquage des banques". Ni plus ni moins ! Quant à la société que fréquente notre avocat, elle semble se diviser en auteurs et en "maîtresses de maison", lesquelles ne peuvent pas sérieusement revendiquer le titre des premiers, condamnées qu'elles sont à n'écrire que des vers sans espoir de les publier...

A lire cet avocat, les exceptions traditionnelles en faveur de la citation et de la copie privée ne seraient plus qu'un "mirage" auquel s'attachent encore quelques esprits naïfs, et les œuvres tombées dans le domaine public pourraient même bientôt être taxées au profit d'auteurs vivants. Rançonner Molière et Balzac pour payer une rente à l'auteur de L'Alchimiste ? Tout est possible. On a bien envisagé de taxer les disques durs, sans considération de leur contenu, au profit d'une nébuleuse d'auteurs... Mieux encore, selon M. Pierrat, l'univers entier serait appelé à se transformer en un immense bazar où la moindre fontaine publique ne pourrait être reproduite sans qu'une société de gestion perçoive sa dîme, pour le plus grand profit des bureaux d'avocats. Heureusement, certains jugements récents, comme celui du tribunal de grande instance de Lyon sur les colonnes de Buren [1], permettent de croire qu'il y aurait des limites au mouvement de privatisation dont $\mathrm{M}$. Pierrat se fait le champion.

Laissant là ce discours, je m'attacherai plutôt à celui de Pierre-Yves Gautier, qui, tout en partageant globalement les vues du premier, présente au moins l'intérêt d'aborder ces questions sur un ton nuancé et favorable à la discussion.

Pierre-Yves Gautier commence par signaler "l'extrême dépendance" de 
l'internaute à l'égard des moteurs de recherche. De fait, en rendant visible et accessible toute la richesse du Web, ces nouveaux outils en ont fait l'auxiliaire privilégié de la mémoire pour une part croissante de la population. Le moteur de recherche est à la lecture sur écran ce qu'un ouvrage en édition Pléiade est à la lecture sur papier : alors que la seconde est essentiellement continue et déterminée par les stratégies narratives de l'auteur, la première est éminemment fragmentée et soumise aux buts du lecteur. Loin d'affirmer que l'internaute ne "lit" pas, je maintiens qu'il ne lit ni romans, ni gros traités sur son écran d'ordinateur, mais écume divers médias ou interroge des bases de données afin d'obtenir des réponses précises à ses questions. Auxiliaire de plus en plus "intelligent", le moteur de recherche encourage ainsi une lecture ergative, c'està-dire orientée vers le travail (en grec : ergon) et qui s'oppose à la lecture loisir ou à la lecture méditation -- cette précision terminologique étant ici donnée à l'intention de M. Pierrat qui, comme le capitaine Haddock, tend à prendre pour des insultes les mots rares qu'il vient tout juste de découvrir. Il est à prévoir que cet outil va avoir sur nos structures conceptuelles et notre rapport à la culture des répercussions comparables en importance à celles qu'a eues le passage du rouleau de papyrus au codex et à l'imprimerie.

Le juriste avoue ne pas comprendre "la différence qui devrait être faite entre la place de cinéma que l'on paie, ou le ticket de musée ou le livre acheté en librairie". Tout en contestant l'assimilation pure et simple du monde physique et du monde virtuel, ainsi que la mise sur un même plan de ces trois types de consommation culturelle, je rappelle que, lorsqu'on va au théâtre, le prix payé dépend souvent de la place occupée, car celle-ci permet une appréhension de l'œuvre de plus ou moins bonne qualité. De même, dans une librairie ou une bibliothèque, on ne vous fera pas payer pour avoir simplement feuilleté des livres et bouquiné à votre aise. Le monde physique permet ainsi de départager les usages payants et ceux qui ne le sont pas, grâce à l'appropriation d'un objet matériel, dans le cas du livre, ou en établissant des tarifs dégressifs selon la qualité de la consommation effectuée dans un espace donné. Il a donc été jusqu'ici relativement facile aux textes de loi de maintenir le principe d'un droit d'auteur intangible : l'auteur étant séparé du lecteur par deux catégories d'intermédiaires, l'éditeur et le libraire, ce sont ces derniers qui assurent l'espace de jeu nécessaire au bon fonctionnement du système. En outre, contrairement à ce que laissent entendre nos deux juristes, les exceptions au principe de rétribution obligatoire et automatique de l'auteur ne manquent pas, mais elles sont devenues invisibles sous la force de la coutume. Ainsi, il n'y a pas de droits versés sur l'achat d'un livre d'occasion: cette entorse à un principe présenté comme absolu ne semble pas avoir préoccupé $\mathrm{M}$. Gautier, qui nous convie en toute bonne foi au Boulevard Saint-Michel, sans prendre garde au paralogisme dans lequel il tombe! Dans le monde physique, le propriétaire d'un ouvrage peut 
ainsi l'échanger, le revendre ou en faire cadeau, sans devoir obtenir l'autorisation du détenteur des droits. Or, cette libre disposition des objets légalement acquis est sérieusement limitée dans le monde virtuel, où un même produit numérique ne sera souvent pas transférable d'un ordinateur à un autre en raison des dispositifs anticopie qui enlèvent à son légitime propriétaire une jouissance pleine et durable.

Même si le monde virtuel offre une gamme de comportements beaucoup moins étendue que le monde physique, il est possible aussi d'y observer des différences très nettes dans l'appropriation qui est faite des textes. Ainsi, l'internaute qui veut lire attentivement un article va souvent l'imprimer : la procédure est tellement courante que la plupart des médias offrent une version préformatée à cette fin. L'univers numérique n'est donc pas une masse indifférenciée et justiciable d'un traitement uniforme. Divers éditeurs travaillent d'ailleurs à mettre au point des logiciels susceptibles d'assurer une lecture confortable sur écran ou sur tablette numérique. Par exemple, le eBook Reader permet à l'usager de surligner des passages, d'ajouter des notes en bas de page ou de consulter automatiquement un dictionnaire pour préciser le sens d'un mot. Ce sont là des procédures d'appropriation qui correspondent à une lecture de fond et non au survol rapide auquel se réduit souvent la lecture sur écran, quand le texte est débité au kilomètre. La consultation d'un fragment de texte dans une base de donnée est aussi une opération très différente de la lecture de l'œuvre intégrale : relevant davantage du régime de la citation que de celui de la lecture suivie, elle mériterait de se voir reconnaître par la loi le même statut d'exception.

Si les différences dans l'appropriation et l'usage des textes sur écran ne justifiaient pas à elles seules un régime juridique différent, il faudrait encore considérer l'intérêt pour la société de rendre accessible sur le Web la plus grande part de ses richesses culturelles et du savoir accumulé afin de permettre l'épanouissement de l'ensemble des citoyens. Dans un monde en voie de complexification croissante, il sera en effet bientôt impossible de participer de plein pied à la vie sociale sans avoir accès à d'énormes quantités de données. L'information, et la capacité d'y accéder, sera alors un bien de première nécessité. Le ministère de l'Éducation a certainement un rôle de premier plan à jouer dans ces domaines, et il aurait intérêt à étudier de près l'exemple donné aux États-Unis par le MT.

Naturellement, une action en faveur du libre flot de l'information suppose une mise à distance de l'idéologie néolibérale qui imprègne aujourd'hui toute la pensée sociale. La chose est d'autant plus difficile que la spéculation qui a accompagné l'expansion du commerce électronique a donné cours à une vaste utopie marchande où les productions culturelles recyclées par les géants du divertissement constitueraient une inépuisable source de richesse. Las ! Le 
recouvrement de la sphère culturelle par la sphère marchande n'entraînera pas une nouvelle Renaissance. Au contraire, en voulant à tout prix "optimiser le rendement des œuvres", selon le jargon de M. Pierrat, on risque de créer une société orpheline de sa culture. On limite aussi le jeu de la création, celle-ci dût-elle prendre la forme du recyclage et de la réinterprétation de productions antérieures, comme dans le cas des Misérables. Racine et Molière n'avaient-ils pas puisé librement dans la source antique?

Encore une fois, il ne s'agit pas de "nier" le droit d'auteur ou la propriété intellectuelle, mais de dénoncer l'usage qui en est fait par les grands monopoles en vue de limiter le domaine public et de cadenasser le Web. L'apparition d'un nouveau média, tout en aiguisant les appétits, prend au dépourvu le corps législatif et juridique, qui peut errer dans ses décisions de façon spectaculaire. Dans une thèse fort documentée sur l'histoire du droit d'auteur, Laurent Pfister rappelle ainsi que, peu de temps après l'invention de l'imprimerie, la République de Venise avait adopté un décret interdisant "à toute personne autre que l'imprimeur allemand Johannes von Speyer d'imprimer des livres [2]" ! Le brevet accordé aux États-Unis au processus de commande par simple clic d'Amazon relève d'une semblable précipitation [3] .

Vouloir faire échapper les textes à leur "prison de papier" n'équivaut pas à considérer le droit d'auteur comme une prison. Seul le papier est visé ici, engoncé dans la rigidité du monde physique, par opposition à la fluidité du texte numérisé sur le Web, qui est intégralement indexé, accessible à tous et de partout, et susceptible d'entrer en résonance avec tout ce qui porte sur le même sujet. La proposition mise de l'avant est d'assurer aux œuvres écrites une durée de protection à deux paliers, afin qu'elles soient accessibles virtuellement sur le Web après un laps de temps beaucoup plus court que celui qui est en vigueur pour les œuvres imprimées, tout en maintenant la possibilité d'une intervention éditoriale protégée. Est-ce là une si grande hérésie ? Si la loi a systématiquement étendu, au cours des deux derniers siècles, la durée du droit d'auteur, en quoi serait-ce une "expropriation forcée" que d'amorcer aujourd'hui un mouvement inverse en faveur du monde virtuel, afin que le principe millénaire de la bibliothèque puisse être sauvegardé et jouer pleinement son rôle ? Le progrès va-t-il nécessairement dans le sens de la disparition de l'espace public ? On a beau dire que "70 ou 95 ans ne sont pas une éternité" : à toute fin pratique, cela exclut une œuvre des moteurs de recherche et de la consultation en ligne durant trois ou quatre générations, ce qui est plus que suffisant pour introduire une cassure dans les habitudes de consommation culturelle.

$\mathrm{Au}$ lieu de voir l'avenir comme allant nécessairement dans le prolongement linéaire d'un mouvement amorcé depuis quelques siècles, et donc de croire avec M. Pierrat que, bientôt, la durée du droit d'auteur s'étendra sans aucune 
limitation de génération en génération, il est essentiel de reconnaître la rupture que représente l'arrivée de l'Internet et de l'espace numérique. Tout comme il a fait sa place au texte imprimé voilà plus de cinq siècles, le droit doit faire la sienne à ce nouveau médium, dont la fluidité n'est pas sans analogie avec celle de la parole, l'Internet étant la place publique du XXIe siècle, l'espace où circule la rumeur du monde. Dans la société orale qui précédait Gutenberg, la notion de propriété intellectuelle ne pouvait guère avoir de base matérielle pour la bonne raison que les œuvres échappaient par définition à leur auteur : circulant oralement, elles étaient bientôt sur toutes les lèvres, colportées par des bardes et des troubadours qui se faisaient un répertoire rapiécé de toutes parts. Avec l'imprimerie tout change. Il est alors possible d'établir sans contestation la date de publication d'une œuvre et d'en suivre les avatars à la trace. Grâce au support physique du papier, la perception d'une redevance pourra devenir normale et facile d'application, même si le cadre juridique du droit d'auteur exigera plus de trois siècles pour être mis en place. Bref, c'est l'imprimerie qui a donné une forme à la propriété intellectuelle et qui a permis à ce qui n'était d'abord qu'un réseau métaphorique de prendre corps sous la forme d'une législation universelle. Étendre celle-ci au monde virtuel, sans aucun espace de jeu, ne pourra pas se faire sans restreindre dangereusement les libertés individuelles ou étrangler le nouveau média qu'est l'Internet. C'est aussi priver les auteurs de la notoriété que peut leur donner la possibilité d'une libre consultation en ligne.

La combinaison de l'ordinateur avec des systèmes de cryptage donne aujourd'hui aux détenteurs de droits un contrôle sur les usagers dont Victor Hugo n'aurait pas pu rêver. Il est en effet possible de contrôler l'accès de l'usager à tout produit culturel numérisé, d'en limiter la durée, la fréquence et l'étendue dans le temps. Le consommateur culturel ainsi fiché et parqué dans les bases de données de Microsoft ou Vivendi n'est pas loin de ressembler à un cheptel dont l'impératif ultime serait de consommer en silence, sous le contrôle inviolable des dispositifs de protection. Dans le numéro d'octobre 2001 de la revue Wired, J eff Howe démontre de façon saisissante les dérives de la licence numérique en transposant celle-ci dans le monde du papier : le "Reader license agreement" qui en résulte constitue une véritable pièce d'anthologie qui devrait être étudiée dans toutes les facultés de droit. Cet article très au fait des aspects juridiques et technologiques cite les propos de Pam Samuelson, bien placée pour observer cette dérive en tant que directrice du Centre de droit et technologie de Berkeley. Selon elle nous serions "sur la voie d'un nouveau féodalisme, car [à l'époque féodale] les seigneurs pensaient qu'ils avaient le droit de tout contrôler et que tous les autres n'avaient que des droits d'usage" (p. 147).

Pierre-Yves Gautier dit fort justement que le droit est "source permanente d'équilibre entre des intérêts antagonistes". Mais pour atteindre à un juste 
équilibre, il faut qu'il y ait débat, précisément, et que le point de vue des usagers puisse se faire entendre. Il faut aussi développer dans les cours de justice et les facultés de droit une réflexion juridique et philosophique soucieuse de faire sa place au domaine public, car celui-ci est plus nécessaire que jamais dans une époque de fragmentation sociale.

Par ailleurs, il ne faudrait pas se réfugier trop facilement derrière l'exception française. La mondialisation joue aussi dans le domaine du droit et tend à forcer les juridictions nationales à s'ajuster sur le plus petit dénominateur commun tout en donnant le champ libre aux lobbies. On l'a vu encore en juin dernier lors de la Conférence de La Haye sur l'harmonisation des régimes de droit international privé : les États-Unis, qui avaient d'abord demandé que la propriété intellectuelle soit exclue de la convention, ont modifié leur position sous la pression de Time AOL/Warner, Disney, la Motion Picture Association et la Recording Industry Association of America [4]. Là encore, ce sont les très grands groupes qui exercent le plus de pressions sur les législateurs et non des auteurs individuels.

De même, il faut questionner les propos lénifiants de Pierre-Yves Gautier sur la nouvelle directive européenne en matière de droit d'auteur adoptée en mai 2001, car celle-ci est loin de présenter une solution satisfaisante aux problèmes que pose le passage à une société digitale, notamment en ce qui concerne la copie privée. Selon l'analyse qu'en a faite Séverine Dusollier pour le site "Droit et nouvelles technologies", "la copie numérique [à titre privé] se voit reconnaître ici un statut purement supplétif dans l'attente d'une généralisation des dispositifs anti-copie qui pourraient, à terme, enlever toute pertinence à cette question" [ $[$ ] . La même chercheuse juge aussi indispensable de "préciser légalement les limites des protections techniques compte tenu de leur effet d'appropriation éventuelle du domaine public et de la restriction de l'accès à l'information qu'elles impliquent" []].

Bref, il y a amplement matière à réflexion pour le Conseil supérieur de la propriété littéraire et artistique que le Gouvernement français a mis en place le 11 mai 2001 et qui doit fonctionner comme une instance de médiation en ces matières. Il faut espérer que cet organisme contribuera à la création d'un "espace public numérique dense et ouvert", selon les propres mots de la ministre de la Culture, et qu'il saura prendre en compte les besoins des usagers et de la société dans son ensemble. Pour ma part, je suis d'un optimisme très modéré.

\section{Notes}

[1] L'architecte Daniel Buren poursuivait une société éditrice de cartes postales au motif qu'elle avait reproduit sans autorisation ses colonnes sur la place des 
Terreaux à Lyon. Le tribunal a débouté l'architecte. On lira là-dessus l'article de Gérard Monnier, dans Le Monde du 18 avril 2001, qui commente favorablement ce jugement et s'oppose très nettement à la position de Pierre-Yves Gautier, publiée dans le même journal quelques jours auparavant, en rappelant notamment que Gustave Eiffel avait explicitement renoncé à ses droits sur sa tour. Nul doute que cette dernière décision doit paraître aberrante à mes deux contradicteurs, pour qui la notion d'un espace public de la culture sonne sans doute comme un oxymoron.

[2]"L'auteur, propriétaire de son œuvre? La formation du droit d'auteur du XVIe siècle à la loi de 1957", Thèse de doctorat en droit, Université Robert Schuman, Strasbourg, 1999, p. 41.

[3] Cette décision a été abondamment critiquée, notamment par Tim O'Reilly pour qui "the Amazon 1-Click Patent is one more example of an "intellectual property" milieu gone mad ." http://www.oreilly.com/ask_tim /amazon_patent.html

[4] http:// www.cyberie.qc.ca/ chronik/ 20010703.html\#b

[5] http:// www.droit-technologie.org/ fr/ dossiers/internet_et_droit_auteur.pdf, p. 17.

[6] ibidem, p. 20. 